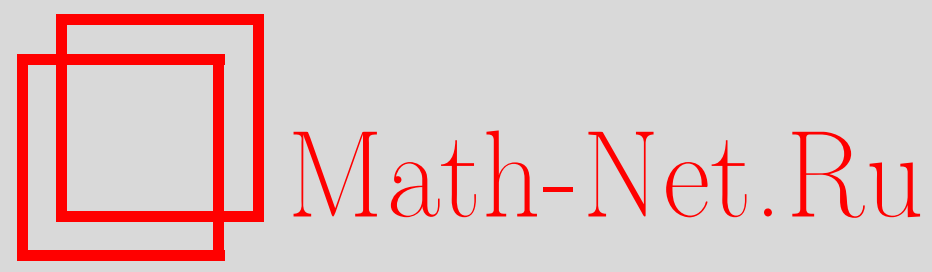

А. Н. Максименко, Диаметр ридж-графа циклического многогранника, Дискрет. матем., 2009, том 21, выпуск 2, 146152

DOI: https://doi.org/10.4213/dm1054

Использование Общероссийского математического портала Math-Net.Ru подразумевает, что вы прочитали и согласны с пользовательским соглашением http://www . mathnet.ru/rus/agreement

Параметры загрузки:

IP : 54.198.67.100

26 апреля 2023 г., 11:23:30 
УДК 519.1

\title{
Диаметр ридж-графа циклического многогранника
}

\author{
() 2009 г. А. Н. Максименко
}

\begin{abstract}
Показано, что диаметр ридж-графа, то есть графа многогранника $C^{*}(d, n)$, двойственного к данному циклическому многограннику, где $d$ - размерность, а $n$ - число фасет, равен $n-d-\max \{0,\lceil(n-2 d) /(\lfloor d / 2\rfloor+1)\rceil\}$.
\end{abstract}

Следуя общепринятой терминологии $[1,2,10], d$-мерный многогранник будем называть $d$-многогранником, а его $k$-мерные грани $-k$-гранями. Соответственно, 0 -грани называются вершинами, 1-грани - ребрами, $(d-1)$-грани $d$-многогранника называются фасетами (или гипергранями), $(d-2)$-грани называются субфасетами (или риджами). Две вершины многогранника называются смежными, если они соединены ребром многогранника. Граф многогранника есть совокупность вершин и ребер многогранника. Множество вершин ридж-графа многогранника совпадает с множеством фасет и две фасеты смежны, если они имеют общий ридж. Таким образом, ридж-граф многогранника является, по существу, графом двойственного к данному многогранника $[1,2]$.

Путь в графе есть последовательность вершин $\left(v_{0}, v_{1}, \ldots, v_{l}\right)$, где каждые две соседние вершины $v_{i-1}$ и $v_{i}, i \in\{1, \ldots, l\}$, смежны и число $l$ есть длина пути. Расстоянием между двумя вершинами в графе называется длина кратчайшего соединяющего их пути. Диаметр графа - это расстояние между двумя его наиболее удаленными друг от друга вершинами.

Обозначим через $\Delta(d, n)$ наибольший диаметр графа многогранника в классе всех $d$-многогранников с $n$ фасетами. В 1957 г. В. М. Хирш предположил [5], что $\Delta(d, n) \leqslant n-d$ (точнее, предположение было высказано для всех полиэдров). Вопрос о справедливости этой гипотезы в общем случае до сих пор остается открытым. Известные верхние оценки для $\Delta(d, n)$ не являются даже полиномиальными [12]. Из работ [7, 11] известно, что для $n>d \geqslant 8$ справедливо неравенство $\Delta(d, n) \geqslant n-d$. Известно $[2,10]$, что гипотезу достаточно доказать для простых многогранников (каждая вершина таких $d$-многогранников принадлежит ровно $d$ фасетам). Кроме того, имеется ряд частных результатов, связанных с вычислением диаметров многогранников из некоторых специальных классов [2, 12]. Одними из наиболее известных и важных классов многогранников являются циклические $[4,8]$ и двойственные к ним многогранники.

Циклическим многогранником $C(d, n)$ называется многогранник с $n$ вершинами $x^{i}=x\left(\tau_{i}\right), i \in\{1, \ldots, n\}$, лежащими на кривой $x(\tau)=\left(\tau, \tau^{2}, \ldots, \tau^{d}\right)$ в пространстве $\mathbf{R}^{d}$. Уникальность циклических многогранников определяется рядом свойств [2]. Они симплициальны, $\lfloor d / 2\rfloor$-смежностны и имеют максимальное число граней всех размерностей в классе $d$-многогранников с фиксированным числом вершин [17]. Соответственно, двойственные к циклическим многогранники $C^{*}(d, n)$ являются простыми и имеют 
максимальное число вершин в классе $d$-многогранников с фиксированным числом $n$ фасет. Эти обстоятельства вселяли надежду на то, что, кроме всего прочего, $C^{*}(d, n)$ станут примером многогранников с наибольшим диаметром графа. К сожалению, уже для $d=4$ и $n=9$ их диаметр равен 4, в то время как в работе [15] приводится пример 4-многогранника с тем же числом фасет и диаметром графа равным 5 (позднее было показано [3], что это единственный, с точностью до комбинаторной эквивалентности, для данных $d=4$ и $n=9$ пример многогранника с диаметром 5). Введем обозначение

$$
\Delta_{c}(d, n)=\operatorname{diam} C^{*}(d, n) .
$$

В 1964 году В. Кли показал [13], что гипотеза Хирша справедлива для $C^{*}(d, n)$ :

$$
\Delta_{c}(d, n) \leqslant n-d
$$

а при $d<n \leqslant 2 d$ в (1) выполняется равенство. Там же было высказано предположение о том, что для $n>2 d$ выполнено равенство $\Delta_{c}(d, n)=\lfloor n / 2\rfloor$ (чуть позже было показано [15], что оно неверно). Но даже эти результаты, ввиду малой доступности работы [13], настолько плохо известны, что по прошествии более 30 лет делаются попытки нахождения величины $\Delta_{c}(d, n)$ для $n=2 d$ с помощью вычислительной техники [6], а в работе [16] при упоминании факта (1) приводится ссылка на работу [14], не содержащую этого результата.

Для того чтобы поставить точку в этом вопросе, ниже приводится точное значение для $\Delta_{c}(d, n)$.

Теорема 1. Диаметр $\Delta_{c}(d, n)$ ридж-графа цииклического многогранника $C(d, n)$ вычисляется по формуле

$$
\Delta_{c}(d, n)= \begin{cases}n-d & \text { при } d<n \leqslant 2 d, \\ n-d-\lceil(n-2 d) /(\lfloor d / 2\rfloor+1)\rceil & \text { nри } n>2 d .\end{cases}
$$

Доказательство. Справедливость теоремы для $d<n \leqslant 2 d$ доказана в [13], поэтому далее будем предполагать, что $n>2 d$.

Обозначим множество вершин многогранника $C(d, n)$ через $X=\left\{x^{1}, \ldots, x^{n}\right\}$, предполагая, что вершины пронумерованы в порядке возрастания параметра $\tau$, то есть $\tau_{1}<\ldots<\tau_{n}$.

Справедлив следующий критерий Гейла [9]. Все грани многогранника $C(d, n)$ являются симплексами и подмножество $Y \subset X$, состоящее из $d$ вершин, определяет некоторую фасету тогда и только тогда, когда для любых двух $x^{i}, x^{j} \in X \backslash Y, i<j$, число вершин $x^{k} \in Y$, для которых $i<k<j$, четно.

В частности, из симплициальности циклического многогранника следует, что две его фасеты смежны, если они имеют ровно $d-1$ общих вершин.

Проведем доказательство теоремы для случая четной размерности. Пусть $d=2 k$, покажем, что

$$
\Delta_{c}(d, n) \leqslant n-d-(n-2 d) /(k+1),
$$

и приведем пример двух фасет циклического многогранника, расстояние между которыми равно целой части от верхней границы (2).

Из критерия Гейла следует, что вершины каждой фасеты можно единственным образом разбить на $k$ непересекающихся пар вида $\left\{x^{i}, x^{i+1}\right\}$, где $i \in\{1, \ldots, n\}$ и сложение 

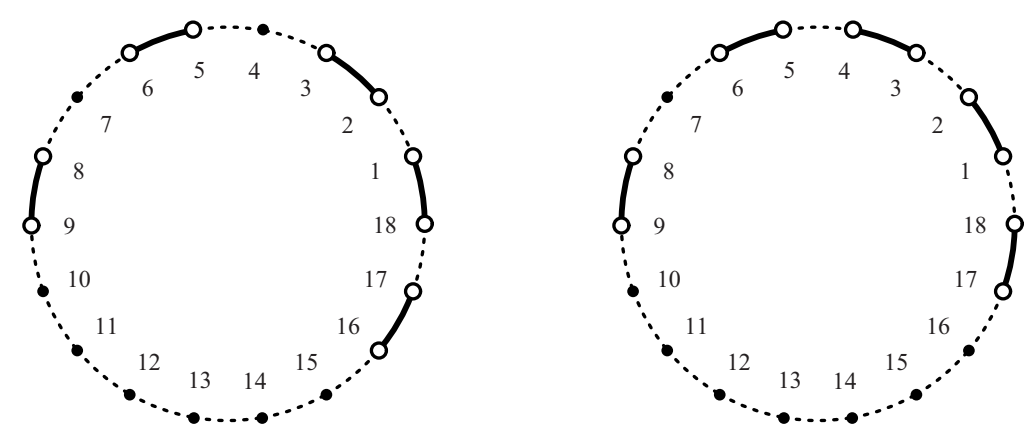

Pис. 1. Две смежные фасеты многогранника $C(10,18)$

$i+1$ проводится по модулю $n$ (если $i=n$, то $i+1=1)$. И, наоборот, любое подмножество $Y \subset X$ вида $Y=\left\{x^{i_{1}}, x^{i_{1}+1}, x^{i_{2}}, x^{i_{2}+1}, \ldots, x^{i_{k}}, x^{i_{k}+1}\right\}$ есть множество вершин некоторой фасеты.

Для наглядности дальнейшего изложения каждой вершине $x^{i}$ поставим в соответствие точку $v_{i}$ на окружности единичного радиуса

$$
v_{i}=(\cos (2 \pi i / n), \sin (2 \pi i / n)
$$

Две точки $v_{i}$ и $v_{i+1}$ будем называть парой и обозначать $p_{i}$, множество всех таких пар для $i \in\{1, \ldots, n\}$ обозначим $P$.

Предположим, что вершины некоторой фасеты заданы набором непересекающихся пар. Тогда переход к смежной фасете означает сдвиг одной или нескольких примыкающих друг к другу пар на одну точку вдоль окружности (см. рис. 1). Иными словами, чтобы перейти к любой смежной фасете, достаточно выбрать одну из $k$ пар и направление смещения (по часовой или против часовой стрелки), при этом, если на пути смещения пары находится другая пара, то она смещается в том же направлении, что и первая, и т. д.

Далее будем пользоваться следующим правилом смещения: при смещении пара может одновременно продвигать и несколько примыкающих соседей, лежащих у нее на пути, но не может пересекаться с другой парой.

Итак, задача вычисления расстояния между фасетами в ридж-графе циклического многогранника сводится к следующей. На окружности с $n$ точками выбраны $k$ пар точек, определяющих вершины первой фасеты, далее называем их фишками, и отмечены $k$ пар, соответствующих вершинам второй фасеты, далее называем их ячейками (см. рис. 2). Требуется найти наименьшее число правильных смещений фишек, достаточное для того, чтобы все ячейки были заняты фишками. Обозначим это число $l\left(F_{1}, F_{2}\right)$, здесь $F_{1}-$ множество фишек, а $F_{2}$ - множество ячеек, $F_{1}, F_{2} \subset P$.

Каждой паре из множества $P \backslash\left(F_{1} \cup F_{2}\right)$ поставим в соответствие дугу окружности между точками $v_{i}$ и $v_{i+1}$, не содержащую самих этих точек. Назовем такую дугу промежутком (см. рис. 2) и будем обозначать $a_{i}$. Множество всех промежутков обозначим через $A$. Очевидно, что $|A|>0$. Подмножество $W \subseteq A$ назовем пустотой, если найдутся $i, j \in\{1, \ldots, n\}$ такие, что $W=\left\{a_{i}, a_{i+1}, \ldots, a_{j}\right\}$, причем $a_{i-1}$ и $a_{j+1}$ не принадлежат $A$. Здесь, как и ранее, при вычислении индексов сложение проводится по модулю $n$. Ясно, что $A$ представимо единственным образом в форме

$$
A=W_{1} \cup W_{2} \cup \ldots \cup W_{s}, \quad 0<s \leqslant d,
$$




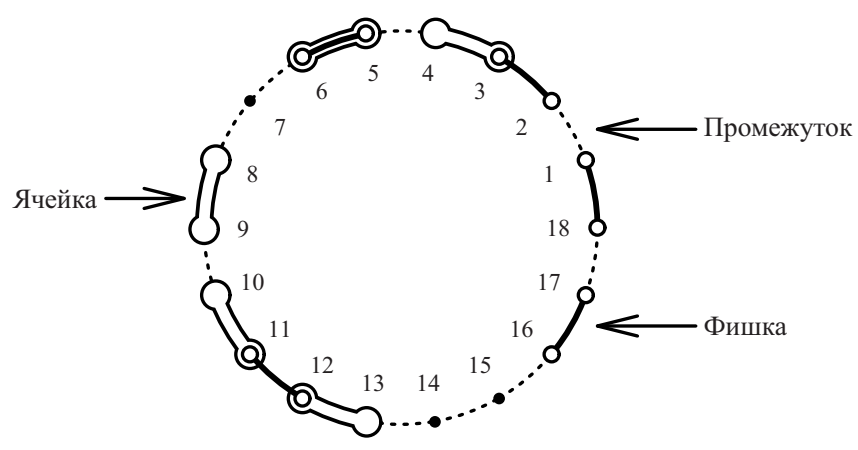

Рис. 2. Пример фасет $F_{1}=\{1,2,3,5,6,11,12,16,17,18\}$ (фишки) и $F_{2}=\{3,4,5,6,8,9,10,11,12,13\}$ (ячейки) многогранника $C(10,18)$

где $W_{i}, i=1, \ldots, s,-$ пустоты.

Определим длину $l(W)$ пустоты $W$ как число точек $v_{i}, 1 \leqslant i \leqslant n$, содержащихся внутри нее, то есть $l(W)=|W|-1$. Тогда суммарная длина пустот равна числу вершин, не принадлежащих ни одной из двух выбранных фасет, и

$$
\sum_{i=1}^{s} l\left(W_{i}\right) \geqslant n-2 d .
$$

Лемма 1. Предположим, что из окружности вырезана пустота $W$ вместе с содержащимися внутри нее точками и фишкам из $F_{1}$ запрещено передвигаться через эту пустоту. Тогда

$$
l\left(F_{1}, F_{2}\right) \leqslant n-d-l(W) .
$$

Доказательство. При каждом правильном смещении от фишек освобождается ровно одна точка, и ровно одна точка становится занятой. Заметим, что при оптимальном передвижении фишек однажды освободившаяся точка не может быть занята вновь. Чтобы убедиться в этом, предположим противное. Тогда возможны два варианта:

(1) Фишка, освободившая точку, заняла ее вновь. Но тогда неоптимальность перемещений очевидна.

(2) Фишка, занявшая точку, сместилась в том же направлении, что и фишка, освободившая эту точку. Но тогда эти два смещения (освобождение и занятие данной точки) можно было бы сделать за один шаг.

Таким образом, число шагов не может превышать числа всех точек, не занятых фишками.

Непосредственно из леммы 1 следует неравенство

$$
l\left(F_{1}, F_{2}\right) \leqslant n-d-\max _{1 \leqslant i \leqslant s} l\left(W_{i}\right) .
$$

В частности, с его помощью легко доказать теорему для $d<n \leqslant 2 d$. Достаточно привести пример двух фасет, имеющих $2 d-n$ общих вершин. Очевидно, что число сближающих смещений для них будет равно $n-d$. 


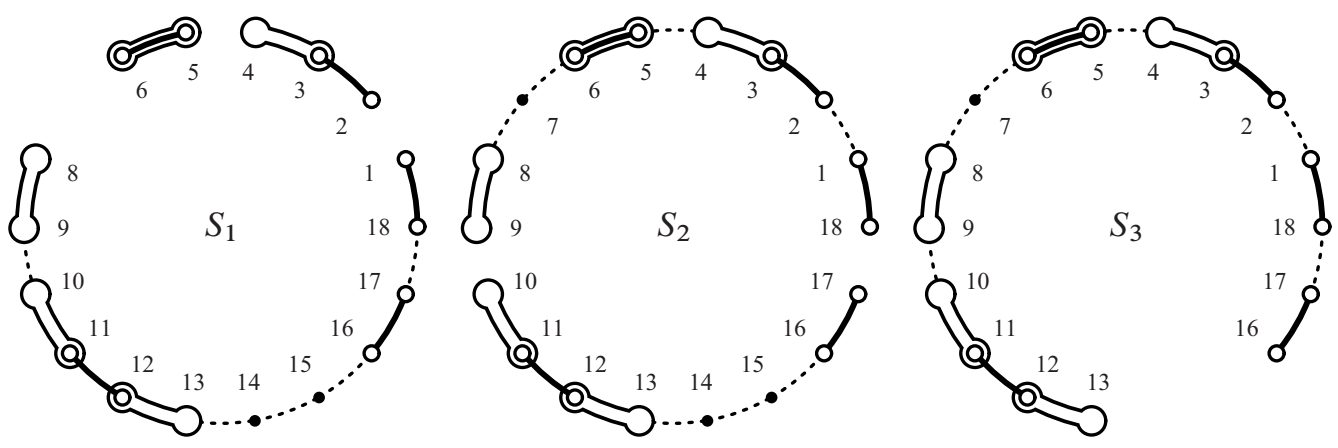

Рис. 3. Три разреза для рис. $2: l\left(S_{1}\right)=1, l\left(S_{2}\right)=0, l\left(S_{3}\right)=2$

Под фразой “между пустотами $W_{i}$ и $W_{j}$ ” будем понимать тот участок окружности, который будет пройден при движении против часовой стрелки от пустоты $W_{i}$ к $W_{j}$. Введем вспомогательную функцию $f(i, j)$, равную числу фишек, расположенных между $W_{i}$ и $W_{j}$, минус число ячеек на этом же участке. Легко заметить, что эта функция обладает следующими свойствами.

Свойство 1. Справедливо равенство $f(i, j)=-f(j, i)$.

Свойство 2. Справедливо равенство $f(i, j)=f(i, m)+f(m, j)$.

Свойство 3 (непрерывность). Если $f(i, j)>1$, то между $W_{i}$ и $W_{j}$ найдется такое $W_{m}$, что $f(i, m)=1$.

Если для некоторых $i$ и $j$ справедливо равенство $f(i, j)=0$, то пустоты $W_{i}$ и $W_{j}$ назовем смежными.

Свойство 4 (транзитивность). Если $W_{i}$ и $W_{j}$ смежны и $W_{j}$ и $W_{m}$ смежны, то $W_{i}$ и $W_{m}$ тоже смежны.

Пусть $W_{i}$ и $W_{j}$ смежны. Тогда, вырезав их из окружности и применив лемму 1 для каждой из двух образовавшихся дуг, получаем неравенство

$$
l\left(F_{1}, F_{2}\right) \leqslant n-d-l\left(W_{i}\right)-l\left(W_{j}\right)
$$

Аналогично мы можем поступить и с несколькими попарно смежными пустотами.

Используя эту возможность, разобьем множество всех пустот на подмножества $S_{i}$, $1 \leqslant i \leqslant t \leqslant s$, так, чтобы все пустоты, принадлежащие одному подмножеству, были попарно смежны и в то же время любые две пустоты из разных подмножеств были бы несмежны. Подмножества $S_{i}$ будем называть разрезами (см. рис.3). Под длиной $l(S)$ разреза $S$ будем понимать суммарную длину входящих в него пустот. Тогда, очевидно,

$$
l\left(F_{1}, F_{2}\right) \leqslant n-d-\max _{1 \leqslant i \leqslant t} l\left(S_{i}\right),
$$

где $t$ - число всех разрезов. Так как

$$
\sum_{j=1}^{t} l\left(S_{j}\right)=\sum_{i=1}^{s} l\left(W_{i}\right) \geqslant n-2 d,
$$




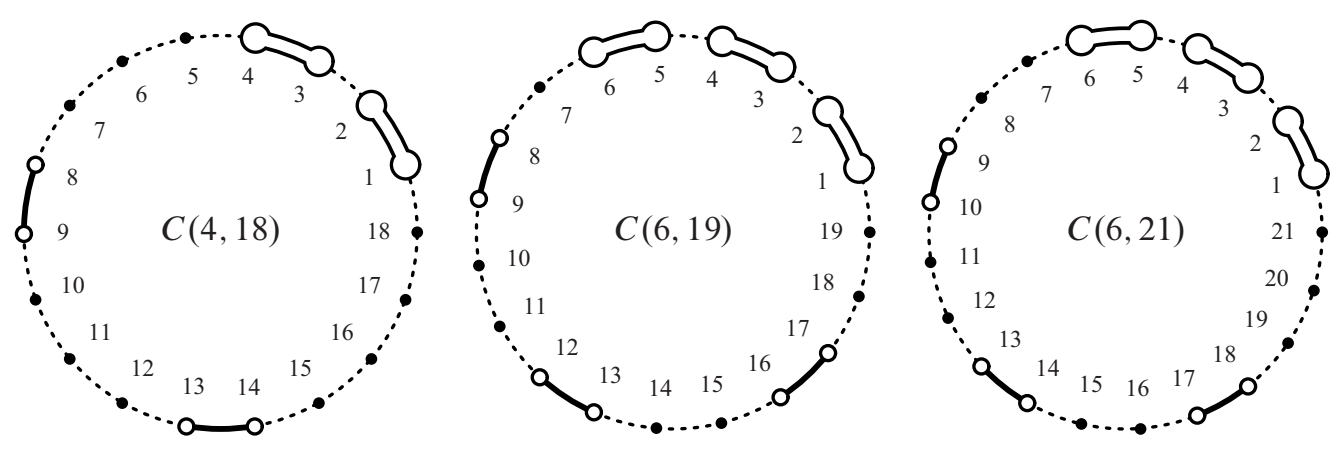

Рис. 4. Примеры диаметрально противоположных фасет

справедливо неравенство

$$
l\left(F_{1}, F_{2}\right) \leqslant n-d-\frac{n-2 d}{t} .
$$

Остается доказать, что

$$
t \leqslant k+1
$$

где $k=d / 2$. Согласно (3), число всех пустот $s \leqslant d$. Поэтому, чтобы установить справедливость неравенства (4), достаточно показать, что среди всех разрезов найдется не более двух, содержащих ровно по одной пустоте.

Пусть разрез $S^{\prime}$ содержит единственную пустоту $W_{i^{\prime}}$. Это означает, что для любого $j \neq i^{\prime}$

$$
f\left(i^{\prime}, j\right) \neq 0 \text {. }
$$

И тогда, в силу свойства 3 (непрерывность), для любого $m \neq i^{\prime} f\left(i^{\prime}, j\right) f\left(i^{\prime}, m\right)>0$, то есть функция $g^{\prime}(j)=f\left(i^{\prime}, j\right)$ для всех $j$ принимает значения одного знака.

Рассмотрим еще один разрез $S^{\prime \prime}$, содержащий лишь одну пустоту $W_{i^{\prime \prime}}, i^{\prime \prime} \neq i^{\prime}$. Покажем, что $f\left(i^{\prime}, j\right) f\left(i^{\prime \prime}, m\right)<0$ для любых $j \neq i^{\prime}$ и $m \neq i^{\prime \prime}$.

Действуя от противного, предположим, что $f\left(i^{\prime}, j\right) f\left(i^{\prime \prime}, m\right)>0$ для некоторых $j$ и $m$; равенство здесь исключается в силу формулы (5) и аналогичного утверждения для $i^{\prime \prime}$. Но тогда это неравенство справедливо при $j=i^{\prime \prime}$ и $m=i^{\prime}$, что невозможно в силу свойства 1. Итак, среди всех разрезов найдется не более двух, содержащих ровно по одной пустоте, и неравенство (4), а вместе с ним и неравенство (2), доказаны.

На рис. 4 приводятся примеры фасет, расстояние между которыми равно наибольшему целому, не превосходящему правой части (2). В [15] тоже содержится один пример такого рода для $d=6$ и $n=23$. Алгоритм построения таких примеров для произвольных $d$ и $n$ ясен из рисунка. Таким образом, для случая четной размерности теорема доказана.

Разберем теперь случай, когда $d=2 k+1$.

Из критерия Гейла следует, что в случае нечетной размерности каждая фасета должна содержать хотя бы одну из двух точек $v_{1}$ или $v_{n}$.

Пусть $F_{1}$ и $F_{2}-$ некоторые фасеты. Возможны два случая.

В первом случае $F_{1}$ и $F_{2}$ содержат одновременно хотя бы одну из точек $v_{1}, v_{n}$. Тогда эту точку можно выбросить из рассмотрения и тем самым перейти к случаю четной 
размерности. Следовательно,

$$
l\left(F_{1}, F_{2}\right) \leqslant(n-1)-(d-1)-\frac{(n-1)-2(d-1)}{k+1}<n-d-\frac{n-2 d}{k+1} .
$$

Во втором случае $F_{1}$ и $F_{2}$ не имеют общих точек в множестве $\left\{v_{1}, v_{n}\right\}$. Не уменьшая общности, предположим, что $F_{1}$ содержит $v_{1}$, но не содержит $v_{n}$, а $F_{2}$ содержит $v_{n}$, но не содержит $v_{1}$. Из критерия Гейла следует, что если мы при очередном смещении освободим $v_{1}$, то обязательно должны занять $v_{n}$, то есть пустота, состоящая из промежутка $a_{n}$, не может входить в какой-бы то ни было разрез. Кроме того, если двигаться против часовой стрелки, то пустота, расположенная правее $v_{1}$ будет смежна пустоте, расположенной левее $v_{n}$. Таким образом, наибольшее число разрезов, как и в случае четной размерности, будет равно $k+1$.

Пример наиболее удаленных друг от друга фасет строится аналогично случаю четной размерности.

\section{Список литературы}

1. Бренстед А., Введение в теорию выпуклых многогранников. Мир, Москва, 1988.

2. Емеличев В. А., Ковалев М. М., Кравцов М. К., Многогранники, графы, оптимизачия. Наука, Москва, 1981.

3. Altshuler A., Bokowski J., Steinberg L., The classification of simplicial 3-spheres with nine vertices into polytopes and non-polytopes. Discrete Math. (1980) 31, 115-124.

4. Carathéodory C., Über den Variabilitätsbereich der Koeffizienten von Potenzreihen, die gegebene Werte nicht annehmen. Math. Ann. (1907) 64, 95-115.

5. Dantzig G. B., Linear programming and extensions. Princeton Univ. Press, Princeton, 1963.

6. Ferrez J.-A., Fukuda K., Liebling T. M., Parallel implementation of graph diameter algorithms. EPFL Supercomputing Review (1998), № 10 3-6.

7. Fritzsche K., Holt F. B., More polytopes meeting the conjectured Hirsch bound. Discrete Math. (1999) 205, 77-84.

8. Gale D., Neighboring vertices on a convex polyhedron. Ann. Math. Stud. (1956) 38, 255-264.

9. Gale D., Neighborly and cyclic polytopes. Proc. Symp. Pure Math. (1963) 7, 225-232.

10. Grünbaum B., Convex polytopes. Wiley, New York, 1967.

11. Holt F. B., Klee V., Many polytopes meeting the conjectured Hirsch bound. Discrete Comput. Geom. (1998) 20, 1-17.

12. Kalai G., Polytope skeletons and paths. In: Handbook of discrete and computational geometry (Goodman J. E. et al., eds.). CRC Press, Boca Raton, FL, 1997, pp. 331-344.

13. Klee V., Diameters of polyhedral graphs. Canad. J. Math. (1964) 16, 602-614.

14. Klee V., Paths on polyhedra. II. Pacific J. Math. (1966) 17, $249-262$.

15. Klee V., Walkup D. W., The $d$-step conjecture for polyhedra of dimension $d<6$. Acta Math. (1967) 133, 53-78.

16. Lagarias J. C., Prabhu N., Counting $d$-step paths in extremal Dantzig figures. Discrete Comput. Geom. (1998) 19, 19-31.

17. McMullen P., The maximum numbers of faces of a convex polytope. Mathematika, Lond. (1970) 17, 179-184. 